\title{
Revised manuscript
}

Published in Colloids and Surfaces A Physicochemical and Engineering Aspects - September 2014 458: 178-186

DOI: $10.1016 /$ j.colsurfa.2014.05.048 


\title{
Tuneable surface modification of PLGA nanoparticles carrying new antitubercular drug candidate
}

\author{
É. Kiss ${ }^{1 *}$, G. Gyulai ${ }^{1}$, Cs. B. Pénzes ${ }^{1}$, M. Idei ${ }^{2}$, K. Horváti $^{3}$, B. Bacsa ${ }^{3}$, Sz. Bősze $^{3}$ \\ 1 Laboratory of Interfaces and Nanostructures, Institute of Chemistry, Eötvös Loránd \\ University, Budapest 112, P.O. Box 32, H-1518 Hungary \\ 2 Office for Research Groups Attached to Universities and Other Institutions \\ of the Hungarian Academy of Sciences, Budapest Nádor u. 7. H-1051 Hungary \\ 3 MTA-ELTE Research Group of Peptide Chemistry, Budapest 112, POB 32, H-1518 \\ Hungary
}

* To whom correspondence should be addressed. Telephone: 36 1-372-2500/1308 Fax: 36 1372-2592 E-mail: kisseva@ chem.elte.hu

\begin{abstract}
Biodegradable poly(lactic-co-glycolic acid)(PLGA 50:50) nanoparticles (NPs) were prepared and characterized in terms of size, composition, zeta potential and colloidal stability. Surface modification of PLGA NPs where primary amino groups were introduced to the Pluronic surface layer was developed. This method allows modulation of the charge character of the nanoparticle surface and provides functional groups for chemical reactions useful for targeting while retaining the aggregation stability of the system. The nanoparticles showed significant interaction with model membrane system (DPPC and DPPC+DPPG lipid layers) depending on the amount and type of Pluronic applied for stabilization of NPs.

A new antitubercular drug candidate was encapsulated into the PLGA NPs. The cellular uptake and the intracellular efficacy against Mycobacterium tuberculosis (Mtb) of the drug and the drug loaded nanoparticulate systems were investigated. These formulations were successfully taken up by MonoMac6 human monocyte cells and highly enhanced the availability and efficacy of the drug against $M t b$ which was demonstrated in comparative in vitro experiments.
\end{abstract}

\section{Keywords}

membrane affinity of NPs; antitubercular drug candidate;PLGA nanoparticles;cellular uptake; surface modification; Pluronic127-amine 


\section{Introduction}

Polymeric nanoparticles (NPs), typically in the size range of 20-300 nm, have attracted great attention in the study of drug delivery systems. Biodegradable polymers like poly(lacticco-glycolic acid) copolymers, PLGAs are the most common to prepare drug carrying nanoparticles. The incorporated drug is physically and uniformly dispersed in the matrix of polymer nanoparticles in most cases. Important advantages associated with the use of these particulate vehicles are the ability to increase solubility of the drug, protect drugs from degradation, controlled and sustained release, and various routes of administration, including oral, nasal, intra-ocular or parenteral [1-3]. Furthermore, they offer a means for the localized or targeted delivery of drugs to specific tissue/organ sites of interest. The beneficial consequences of those properties are the reduced toxicity and considerably limited side effects.

To exploit the advantageous pharmaceutical behaviour of PLGA drug delivery nanoparticles they have to meet the requirements of stability in biological fluids, circulation for a prolonged time in the body without the risk of aggregation [4]. It is known that unwanted capture of colloidal particles by the mononuclear phagocyte system (MPS) can be avoided if the particles are surface modified by the adsorption or grafting of hydrophilic polymers such as polyethylene glycol (PEG). The attached PEG layer provides a steric barrier to the adsorption of plasma components and as a consequence opsonisation is greatly reduced. This steric barrier of PEG coated particles have been shown to present extended circulation time and concurrently generates the colloidal stability [5]. Nanoparticles with stealth properties that can circumvent clearance and defence mechanisms are the most promising formulations [6]. The responsive character of PEG [7] can even be utilized for drug incorporation [8].

Various techniques for formation of PEG layer on PLGA surface were developed. The activation and chemical coupling [9,10] yields a stable layer but is accompanied by partial degradation of the polyester. The PEG-containing triblock copolymer (Pluronic) proved to be an efficient surface modifier which can be applied in a blend form since it is miscible with PLGA in a certain concentration range depending on their composition [11-13]. The adsorption of Pluronic is however, the most simple and straightforward method to coat PLGA nanoparticles [5]. The adsorbed layer is sufficiently stable as it was demonstrated in film experiments [14] and several other studies [15,16].

Considering the nanoparticle drug formulations in clinical trials the anticancer therapy is the most extensively studied and developed area [4,6,8,17]. Many small molecular, anticancer drugs have been encapsulated into PEGylated PLGA nanoparticles and have been evaluated in vitro and in vivo to treat various cancers. On the other hand, only few studies are aimed at developing antibiotic-loaded PLGA nanoparticles [18,19] to improve the treatment of bacterial infections although it offers significant benefit. Since plasma concentrations of most antibiotics need to be constant, sustained release of drugs from PLGA-based nanoparticles could thus potentially improve the treatment efficacy. Moreover, nanoparticles might enhance the intracellular delivery of antibiotics for some intracellular pathogens [20]. Such drug carrying nanoparticles have to enter cells to reach the site of action. The membrane of the cellular targets of therapeutic agents strongly influences the effectiveness of a drug by controlling or modulating such processes as transport, distribution, and accumulation. 
Investigation of the interaction between an active agent or its carrier and the biomembrane is therefore of great interest [2]. The interfacial properties which influence the interactions in biological environments might be conveniently investigated using simplified models of complex cell membranes [21]. The bilayer [22] or lipid monolayer systems are widely used to characterize quantitatively the membrane affinity of bioactive molecules and reveal the resulting structural changes [23-27]. Nevertheless, the interaction of drug delivery NPs with lipid monolayer is rarely investigated although such studies could be effectively used in developing functionalized nanocarrier systems [2,25,28].

In the present work we describe the encapsulation of a new antitubercular drug candidate (TB515) into PLGA nanoparticles. The cellular uptake and the intracellular efficacy against Mycobacterium tuberculosis (Mtb) of the drug and the drug loaded nanoparticulate systems were compared. Applying a new surface modification method we tried to enhance the membrane affinity and cellular uptake of the drug carrying PLGA nanoparticles. This method allows the modification of the charge character of the nanoparticle surface and provides surface functional groups for chemical reactions used for targeting. Meanwhile the colloid stability of the nanoparticulate system is maintained since the polymer structure around the particles is only slightly varied.

\section{Materials and methods}

\subsection{Materials}

Poly(DL-lactic-co-glycolic acid), PLGA with $50 \%$ of lactic and $50 \%$ of glycolic content (Mw: 50,000-75,000), obtained from Sigma-Aldrich, Hungary.

Poly(ethylene oxide)/poly(propylene oxide)/poly(ethylene oxide), PEO-PPO-PEO triblock copolymer, Pluronic 127 (Mw: 12600) (provided by BASF HungariaKft.), was applied as received. The composition of the Pluronic 127 is characterized by the length of the central hydrophobic poly(propylene oxide) (PPO) block (Mw: 3248) and the PEO content (70\%) of the molecule. Amino terminated Pluronic (Pluronic 127-amine) was prepared from Pluronic 127 in two steps. First, terminal hydroxyl groups were modified to aldehyde groups by partial oxidation then to primary amine groups by reductive amination using ammonium chloride and $\mathrm{NaCNBH}_{3}$ [29]. The appearance of terminal amino groups in Pluronic was detected by ninhydrin test. The functionalization yield was above $70 \%$ according to ${ }^{13} \mathrm{C}$ NMR measurements.

1,2-dipalmitoyl-sn-glycero-3-phosphocholine (DPPC) was purchased from Sigma-Aldrich (purity >99\%) and used to form the lipid monolayer in the Langmuir trough. A negatively charged lipid, 1,2-dipalmitoyl-sn-glycero-3-phospho-(10-rac-glycerol) (sodium salt) (DPPG), mixed with DPPC at 25:75\% ratio was applied as the a second model lipid system to mimic a negatively charged natural bacterial lipid bilayer.

TB515 molecule which is a derivative of in silico identified potential antibacterial agent was purchased from Ubichem (Budapest, Hungary) (Figure 1)[30]. TB515 is rather hydrophobic with poor water solubility and estimated $\log P$ of 2.9. Labelling is not needed to detect TB515 in cellular measurements since it is a strong fluorophore. DMSO (Sigma-Aldrich, A.C.S. reagent, $\geq 99.9 \%$ ) was the solvent for TB515 in spectrophotometric measurements.

RPMI-1640 medium, Löwenstein-Jensen medium base and the components of the Sula medium was from Sigma-Aldrich (St. Louis, MO, USA). HPMI buffer contains $9 \mathrm{mM}$ 
glucose, $10 \mathrm{mM} \mathrm{NaHCO} 3,119 \mathrm{mM} \mathrm{NaCl}, 9 \mathrm{mM}$ HEPES, $5 \mathrm{mM} \mathrm{KCl}, 0.85 \mathrm{mM} \mathrm{MgCl}_{2}, 0.053$ $\mathrm{mM} \mathrm{CaCl}, 5 \mathrm{mM} \mathrm{Na} 2 \mathrm{HPO}_{4} \times 2 \mathrm{H}_{2} \mathrm{O}(\mathrm{pH} 7.4)$.

Chloroform (p.a., purity 99.4\%) from Merck, Hungary, was used for preparing the lipid spreading solutions. Dichloromethane (HPLC, purity >99.9\%) from Merck, Hungary, and methanol (HPLC, purity >99.9\%) from LGC Promochem were used for cleaning the Langmuir trough and barrier. $\mathrm{NaCl}$ (p.a., Riedel de Haën) was cleaned by heat treatment at $600{ }^{\circ} \mathrm{C}$ to avoid possible contamination presenting surface activity. Doubly distilled water checked by its conductivity $(<5 \mathrm{mS})$ and surface tension $\left(>72.0 \mathrm{mNm}^{-1}\right.$ at $\left.23 \pm 0.5^{\circ} \mathrm{C}\right)$ was the subphase in the Langmuir balance experiments.

\subsection{Preparation of nanoparticles and drug encapsulation}

PLGA nanoparticles were prepared by the nanoprecipitation method similar to that employed previously $[19,28]$. Briefly PLGA was dissolved in acetone to a concentration of $10 \mathrm{gL}^{-1}$. In the case of drug encapsulation the TB515 was also dissolved in the PLGA solution. The organic solution was added dropwise to an aqueous solution of polymeric stabilizer under magnetic stirring $(500 \mathrm{rpm})$. Nanoparticles were formed and stirring was continued overnight to achieve the complete evaporation of acetone. After evaporation of the organic solvent the particle suspension was centrifuged at $3500 \mathrm{~g}$ for $10 \mathrm{~min}$ to remove the possible polymer aggregates and precipitated drug. The amount of sediment in this step was always less than 5\% of the whole solid content [19]. The suspension of nanoparticles was further purified by centrifugation at $12,000 \mathrm{~g}$ for $15 \mathrm{~min}$ where the supernatant was removed and the pellet containing the particles was redispersed with doubly distilled water. This procedure was repeated four times. The preparation conditions of nanoparticles are summarized in Tables 1 and 2. (The preparation of drug loaded samples without the use of Pluronic stabilizer was not possible due to the uncontrolled aggregation of the particles during formulation.)

For the determination of drug content and encapsulation efficiency the purified nanoparticle suspension was lyophilized. A known amount of the solid particles was dissolved in DMSO. The drug content of the solution was determined spectrophotometrically measuring the absorbance at $357 \mathrm{~nm}$. Relating the measured drug amount to the mass of the solid material the drug content (\%) and encapsulation efficiency (\%) were derived. Each experiment was repeated three times.

\subsection{Characterization of nanoparticles}

Particle size, zeta potential and colloid stability were measured to characterize the nanoparticle systems. Pluronic content of the stabilized PLGA nanoparticles was also determined spectrophotometrically.

Average hydrodynamic size and polydispersity of the particles were determined using a dynamic light scattering (DLS) system (Brookhaven Instruments, USA).The suspensions of nanoparticles were measured at $0.1 \mathrm{gL}^{-1}$ concentration. The recorded autocorrelation functions were analysed by the second order cumulant expansion method. The data were obtained with the average of three measurements.

The Pluronic content of the nanoparticles was determined using the purified suspensions. A colorimetric method based on the complex formation between the ethylene oxide segments of the surfactant and cobalt thiocyanate was adapted [31] and applied in the following way. $3 \mathrm{~g}$ of cobalt nitrate and $20 \mathrm{~g}$ of ammonium thiocyanate was dissolved in and diluted to $100 \mathrm{~mL}$ with doubly distilled water. Volumes of $500 \mu \mathrm{L}$ of a series of standard Pluronic 127 solutions in the concentration range of $0.05-2 \mathrm{~g} \mathrm{~L}^{-1}$ were introduced into $2 \mathrm{~mL}$ centrifuge tubes. $200 \mu \mathrm{L}$ of the cobalt thiocyanate was added to the tubes and the solutions were mixed well. $500 \mu \mathrm{L}$ 
ethyl acetate was then added to the tubes and the solutions were mixed thoroughly. The mixtures were centrifuged at $12000 \mathrm{~g}$ for 1 minute. Three phases separated, an upper ethyl acetate layer containing the bulk of the surplus reagent, an aqueous layer and the deep blue sediment containing the water and ethyl acetate insoluble Pluronic complex. The upper ethyl acetate and aqueous layers were aspirated and discarded. The precipitate was washed with 30 $\mu \mathrm{L}$ of water followed by the aspiration of water. The precipitate was dissolved in $1 \mathrm{~mL}$ of acetone and the absorbance was measured in a spectrophotometer at $624 \mathrm{~nm}$. The resulting calibration curve was used to calculate the Pluronic content of the PLGA nanoparticles.

The PLGA samples were assayed similar to that of the Pluronic standards. Spontaneous aggregation of the particles occurred during the addition of the cobalt thiocyanate reagent. With the addition of the ethyl acetate the PLGA dissolved, so the Pluronic amount determined using the assay represents the total Pluronic content of the nanoparticles. Tests were carried out to check if the presence of PLGA influences the Pluronic concentration determination. The results indicated no interference.

The measurement of electrophoretic mobility of nanoparticles was carried out by means of Malvern Zetasizer 4 apparatus. Smoluchowski approximation was used to calculate zeta potential from mobility values. Nanosuspensions were surveyed in aqueous salt solution $(2 \mathrm{mM} \mathrm{NaCl})$.

The effect of ionic strength on the colloidal stability of PLGA nanoparticles was investigated by adding $\mathrm{NaCl}$ solution to the suspension with increasing concentration. After incubation time of $15 \mathrm{~min}$ absorbance was measured with a spectrophotometer at $400 \mathrm{~nm}$. The concentration of $\mathrm{NaCl}$ where three times higher absorbance was measured comparing to that of the original suspension was used to estimate the critical aggregation concentrations (CAC).

\subsection{Interaction with lipid monolayers}

\subsubsection{Langmuir monolayer studies}

The preparation of the lipid layers and the penetration experiments were carried out using a Langmuir trough, KSV MiniMicro, $\left(5 \times 20 \times 0.6 \mathrm{~cm}^{3}\right)$ with two barriers to provide symmetric film compression. All measurements were performed at $23 \pm 0.5^{\circ} \mathrm{C}$.

For the preparation of the Langmuir layers $50 \mu \mathrm{L}$ of lipid solution $\left(0.2 \mathrm{gL}^{-1}\right)$ was spread onto the aqueous subphase and the solvent was allowed to evaporate for $15 \mathrm{~min}$ before compression. The lipid monolayer was compressed to a given surface pressure (15 or $25 \mathrm{mNm}^{-1}$ ) and at a fixed barrier position aqueous suspension of the polymer nanoparticles was injected below the lipid layer to reach a final concentration of $0.025 \mathrm{gL}^{-1}$ in the subphase. The change in surface pressure as the indicator of interaction of nanoparticles with the lipid layer was recorded as a function of time for 1 or $2 \mathrm{hrs}$. The result of penetration into the monolayer was given as the average of two independent measurements with a deviation $<0.5 \mathrm{mNm}^{-1}$.

\subsubsection{AFM of lipid layers}

Langmuir films were transferred onto a hydrophobized glass plates by the Langmuir-Schaefer technique. The morphology of the lipid monolayer without and with the penetrated nanoparticles was studied with an atomic force microscope (Park Syst. XE-100, South Korea). The AFM scans were conducted at $0.3-0.5 \mathrm{~Hz}$ scanning rate taken typically at scan window of $2.5 \times 2.5 \mu \mathrm{m}^{2}$ using a cantilever NSC15 (Park Syst.) made of $\mathrm{Si}_{3} \mathrm{~N}_{4}$ with a tip curvature radius less than $10 \mathrm{~nm}$. Observations were carried out using noncontact mode in air.

\subsection{Antimicrobial effect and cellular uptake}


2.5.1 Evaluation of antimycobacterial activity

In vitro antimycobacterial activity of the TB515 compound was determined on $M$. tuberculosis $\mathrm{H}_{37} \mathrm{Rv}$ (ATCC 27294) by serial dilution in Sula semi-synthetic medium, which was prepared in-house $(\mathrm{pH} 6.5)$ [30, 32,33]. Compound was added to medium as DMSO solutions at ten various doses (range of final concentration was between 0.5 and $100 \mu \mathrm{g} \mathrm{mL}^{-1}$ ). MIC was determined after incubation at $37^{\circ} \mathrm{C}$ for 28 days. MIC was the lowest concentration of a compound at which the visible inhibition of the growth of $M$. tuberculosis $\mathrm{H}_{37} \mathrm{Rv}$ occurred. In order to confirm the growth inhibition colony forming unit (CFU) was determined by subculturing from the Sula medium onto drug-free Löwensten-Jensen solid medium. Samples were incubated for 28 days.

\subsubsection{Cell culturing}

MonoMac6 human monocytic cell line [34] (DSMZ no.: ACC 124, Deutsche Sammlung von Mikroorganismen und Zellkulturen $\mathrm{GmbH}$, Braunschweig, Germany) was maintained in RPMI-1640 medium containing 10\% FCS, $2 \mathrm{mM} \mathrm{L-glutamine} \mathrm{and} 160 \mu \mathrm{g} \mathrm{m} \mathrm{m}^{-1}$ gentamycin at $37^{\circ} \mathrm{C}$ in $5 \% \mathrm{CO}_{2}$ atmosphere.

\subsubsection{Cellular uptake studies by flow cytometry and fluorescent microscopy}

The measurement of cellular uptake of the PLGA-D1 and PLGA-D2 (Table 2) was evaluated by flow cytometry (BD LSR II, BD Biosciences, San Jose, CA, USA) and fluorescent microscopy (Olympus CKX41, Hamburg, Germany) on MonoMac6 human monocytic cell line. Cells were harvested in the logarithmic phase of growth and plated on a 24-well tissue culture plate $\left(10^{5}\right.$ cells $/ 1 \mathrm{~mL}$ medium/well $) 24$ hours prior to the experiment. PLGA-D1 and PLGA-D2 were dispersed in serum free RPMI medium and running dilutions were prepared. The highest concentration of the compounds on the cells was $20 \mu \mathrm{g} \mathrm{ml}^{-1}$. Cells were incubated with compounds for $3 \mathrm{hrs}\left(37^{\circ} \mathrm{C}, 5 \% \mathrm{CO}_{2}\right.$ atmosphere). After centrifugation (1000 rpm, $5 \mathrm{~min}$ ) supernatant was removed and $100 \mu \mathrm{L} 1 \mathrm{mM}$ trypsin was added to the cells. After $5 \mathrm{~min}$ incubation at $37^{\circ} \mathrm{C} 1 \mathrm{~mL} 10 \%$ FCS/RPMI medium was added then cells were washed two times with medium and resuspended in $0.5 \mathrm{~mL}$ HPMI. The cellular uptake was determined by BD LSR II using $488 \mathrm{~nm}$ (Coherent Sapphire, $22 \mathrm{~mW}$ ) laser. The intracellular fluorescence intensity of the cells was measured on channel PE LP550 (emission at $\lambda=$ $550 \mathrm{~nm}$ ). Data were analyzed with FACSDiva 5.0 software (BD Biosciences, San Jose, CA, USA). All measurements were performed in duplicates. Parallel with flow cytometry measurements microscopic image of the cells were captured with an Olympus CKX41 microscope.

2.5.4 Infection of MonoMac6 monolayers with M. tuberculosis $\mathrm{H}_{37} \mathrm{Rv}$ and determination of efficacy against intracellular bacteria

A modified method based on previous works [35,36] was applied [30]. Briefly, MonoMac6 monocytes $\left(2 \times 10^{5}\right.$ cells $/ 1 \mathrm{~mL}$ medium/well $)$ were cultured with RPMI-1640 medium containing $10 \%$ FCS in a 24-well plate 24 hours prior to the experiment. Adherent cells were infected with $M$. tuberculosis $\mathrm{H}_{37} \mathrm{Rv}$ at a multiplicity of infection (MOI) of 10 for 4 hours. Nonphagocytosed extracellular bacteria were removed and the culture was washed three times with serum free RPMI. The infected monolayer was incubated for 1 day before antitubercular treatment. Infected cells were than treated with TB515, PLGA-D1 and PLGA-D2 at $250 \mu \mathrm{M}$ and $125 \mu \mathrm{M}$ final concentration, respectively. After 3 days the treatment was repeated with fresh solution of the compounds for an additional 3 days. Untreated cells were considered as negative control. After washing steps - in order to remove the antituberculars - infected cells were lysed with $2.5 \%$ sodium dodecyl sulfate solution. The CFU of M. tuberculosis was enumerated on Löwenstein-Jensen solid media after 6 weeks of incubation. 


\section{Results and discussion}

\subsection{PLGA nanoparticles}

Drug loaded or empty polymeric NPs were formed from PLGA by the nanoprecipitation method when a water miscible solvent, acetone was used to dissolve both PLGA and the hydrophobic drug molecule TB515 $(\log P=2.9)$. The details of recepies are summarized in Table 1 and Table 2 for drug loaded samples. Pluronic 127 and its amine derivative Pluronic 127-amine were applied in the aqueous phase as stabilizer in various amounts. Sample 0 was also prepared without stabilizer for comparison.

The NPs obtained were characterized by dynamic light scattering and zeta-potential measurements (Tables 1 and 2). The mean diameter of the Pluronic stabilized NPs was around $150 \mathrm{~nm}$, while without stabilizer somewhat smaller particles were formed with a mean diameter of $139 \mathrm{~nm}$. The particle size was in the same range for drug loaded NPs. All of the samples showed a narrow size distribution with a polydispersity about 0.05 . The PI was below 0.1 even for the bare NPs.

The Pluronic content of the NPs was obtained as $1.0 \pm 0.1 \%(\mathrm{w} / \mathrm{w})$ applying the stabilizer at concentration of $0.1 \mathrm{gL}^{-1}$ (samples 1-3) while $2.2 \pm 0.3 \%(\mathrm{w} / \mathrm{w})$ at $1.0 \mathrm{gL}^{-1}$ Pluronic concentration (samples4-6). Previous analysis performed by XPS technique showed that the surface layer of PLGA particles is rich in Pluronic component [28]. Supposing that the Pluronic is localized on the surface of the NPs the calculated surface concentration of Pluronic is found to be in the range of $0.6-1.3 \mathrm{mg} / \mathrm{m}^{2}$ for the stabilized PLGA NPs. These values are in good agreement with adsorbed amount of Pluronics 0.5 and $1.7 \mathrm{mg} / \mathrm{m}^{2}$ reported on moderately hydrophobic surfaces such as carbon black [37] and hydrophobized silica [38], respectively.

Considering the charge character, the bare NPs presented the highest zeta-potential due to the surface carboxylic groups of PLGA. The absolute value decreased as expected $[4,5,15]$ when the particle surface was covered with Pluronic layer. It is notable, that for samples (no. 4, 5 and 6) when the higher amount of Pluronic was applied the surface charges were more effectively shielded by the polymer chains. The other effect which can also be observed in zeta-potential values is the presence of Pluronic 127-amine in the surface layer (samples no. $2,3,5,6)$ which resulted in further decrease of zeta-potential comparing to the cases when the same amount but unmodified Pluronic 127 was used (samples no. 1 and 4). For the drug loaded NPs the zeta-potential values show the similar influence of the application of Pluronic 127-amine.

The above data suggest that the composition of surface layer formed on the PLGA NPs is in correlation with the type and amount of stabilizer applied in the course of nanoprecipitation process. That allows the design of surface charge character within a given range by varying the ratio of the two Pluronic compounds.

The adsorbed layer of Pluronic on PLGA rendering the surface hydrophilic is responsible for the advantageous protein repellent property and the ability of avoiding RES as it was demonstrated in several works [5,6,38]. At the same time it contributes to the excellent steric stabilization of the NP system. Colloidal stability is generally characterized by the determination of critical aggregation concentration (CAC) [15,39]. The stability of the PLGA NP systems presented in Table 1 was checked by the turbidity measurements. When adding $\mathrm{NaCl}$ electrolyte with increasing concentration to the nanosuspension no detectable aggregation was found up to $1.5 \mathrm{M} \mathrm{NaCl}$ for systems no. 1 to 6 .

The presence of Pluronic provides steric stability to the particles by forming a hydrated layer on the surface. The PLGA NPs without stabilizer (sample 0) however, showed aggregation at $0.03 \mathrm{M} \mathrm{NaCl}$. 
Nanosuspension prepared with Pluronic or Pluronic-amine proved to be similarly stabilized.

Consequently, surface modification with Pluronic 127 and Pluronic 127-amine allows controlling the charge character of the surface in a certain range and offering functional groups for chemical coupling reactions preserving meanwhile the colloid stability of the system.

\subsection{Drug encapsulation}

TB515 drug candidate was encapsulated into the PLGA NPs according to the conditions presented in Table 2. About $1.5 \%$ drug content was achieved with encapsulation efficiency above $30 \%$. The expected maximum drug content calculated from the initial PLGA/drug ratio was $2.5 \%$ for these cases. Increasing the drug loading significantly the drug content could be enhanced close to double but with drastically reduced encapsulation efficiency. Therefore the PLGA-D1 and PLGA-D2 systems were selected for further in vitro studies.

\subsection{Membrane affinity}

DPPC which is the primary phospholipid in the mammalian cell membrane and a significant component of alveolar fluid was chosen to form the lipid monolayer as membrane model. Since the charge is described as important parameter to influence the interaction with cell membrane and govern the cellular uptake $[3,40,41]$ DPPC mixed with DPPG was also used to represent the charged membrane model in the penetration experiments.

The interaction of NPs with lipid layers was studied in Langmuir-balance experiments, where the adsorption and/or penetration of the particles into the lipid layer is the indication of their membrane affinity. The incorporation of NPs into the lipid layer was visualized by AFM after the penetrated layer was transferred to a solid support (Figure 2). The image of corresponding lipid layer without nanoparticle penetration is also shown for comparison. The appearance of particles in the lipid layer is a direct evidence of the interaction since the NPs were introduced into the subphase, and the transfer was performed using the Langmuir-Schaefer (horizontal) technique where the solid support does not come into contact with the subphase.

In order to characterize the degree of interaction, the change in surface pressure was detected following the NP injection below the compressed lipid layer. Penetration curves are plotted and compared for differently stabilized NP systems in Figures 3-5. The concentration of the NPs was the same in all penetration experiments.

Penetration of three types of NPs are displayed in Figure 3. DPPC layer was compressed to $25 \mathrm{mNm}^{-1}$ which is close to the value considered as monolayer-bilayer equivalence pressure.

There is practically no interaction between the bare PLGA particles and DPPC layer, while the Pluronic 127-amine modified PLGA particles resulted in significant and steady surface pressure increase (sample no. 3). The adsorption/penetration of the NPs was greatly increased when the higher amount of stabilizer was applied for surface coating (sample no. 6).

The influence of stabilizer type on the membrane affinity is shown in Figure 4. The initial surface pressure of DPPC film $\left(15 \mathrm{mNm}^{-1}\right.$ in this case) increased due to interaction with Pluronic 127 stabilized NPs (sample no. 4) but the penetration is further improved when the coating was formed from Pluronic 127-amine (sample no. 6). The role of electrostatic interaction in membrane affinity was revealed when penetration of Pluronic 127-amine stabilized NPs (sample no. 3) was compared for DPPC and DPPC+DPPG lipid layers (Figure 5). The above penetration experiments show that Pluronic and Pluronic-amine surface coatings are suitable for substantial enhancement of the interaction with model lipid membrane. 
Since the PLGA NPs are coated with Pluronic block copolymers it is worth to discuss their behaviour taking into consideration the results on corresponding molecular interactions obtained earlier [23]. The adsorption of dissolved Pluronics with various block ratios onto lipid membrane was investigated and Pluronic 127 proved to be the most effective in penetration experiments. The high affinity was explained by the presence of long PPO and PEO chains. It was concluded that the length of PPO block is crucial for the ability to penetrate the lipid monolayer even at high surface pressure. On the other hand, interaction with the lipid molecules can occur in the lipid headgroup region and also some interpenetration of the PEO blocks into the lipid monolayers can contribute to the incorporation.

Improved cellular uptake by application of Pluronics has already been reported for various drug delivery systems. Those include protein modification by Pluronic block copolymers which enhanced intracellular delivery of proteins and brain delivery in vivo [42]. Pluronic also enhanced cellular uptake and nuclear entry of polyplex-delivered DNA [43]. It was a question however, whether Pluronic 127 might help to transfer PLGA NPs into cells and reach the $M$. tuberculosis within the cell. In these in vitro experiments the drug loaded Pluronic and Pluronic-amine coated NPs (PLGA-D1 and PLGA-D2, respectively) were investigated and compared to the effect of pure drug.

\subsection{In vitro experiments}

Antitubercular effect of TB515

The in vitro antimycobacterial activity of the compound was characterized by the determination of the minimal inhibitory concentration (MIC) using M. tuberculosis $\mathrm{H}_{37} \mathrm{Rv}$ culture with 4-week exposure period. TB515 was effective against the bacteria and exhibited the MIC value as $5 \mu \mathrm{g} / \mathrm{mL} ; 19 \mu \mathrm{M}$. The minimum inhibitory concentration of compound TB515 was determined as the lowest concentration that could inhibit visible bacterial growth.

Considering that $M$. tuberculosis is an intracellular pathogen as a host cell (macrophage) model we used human monocytic MonoMac6 cells. MonoMac6 was established as a cell line which appears to have phenotypic and functional characteristic of mature blood monocytes. MonoMac6 cells are attached to the tissue culture plate's well surface without any treatment after hours as a homogeneous cell population. Therefore infected MonoMac6 cell line is an accepted model to determine intracellular activity of antituberculars. Cellular uptake tests were evaluated using the same cell line $[30,35,36]$.

Cellular uptake of TB515 and encapsulated TB515 into PLGA nanoparticles

Cellular uptake was evaluated on MonoMac6 human monocytic cell culture using flow cytometry (BD LSRII, Figure 6) and fluorescent microscopy (Olympus CKX41). In order to rule out the possible contribution of adhesion of the nanoparticles to the MonoMac-6 cellular surface, the uptake rate was evaluated following treatment with trypsin and extensive washing, to remove plasma membrane-bound nanoparticles. The treatment of MonoMac6 monocytic cells with PLGA-D1 and PLGA-D2 has resulted in intense intracellular fluorescence, suggesting that both PLGA-D1 and PLGA-D2 nanoparticles were internalized by the MonoMac- 6 cells, Figure 7. The internalization rates of PLGA-D2 was somewhat higher than the rates of PLGA-D1 formulation, indicating the higher cellular transport potential of PLGA-D2 nanoparticles.

Intracellular killing of $M$. tuberculosis

In vitro activity of new TB515 antitubercular compound on $M$. tuberculosis $\mathrm{H}_{37} \mathrm{Rv}$ culture is valuable $(5 \mu \mathrm{g} / \mathrm{mL} ; 19 \mu \mathrm{M})$, but it is crucial that the compound is efficient against 
intracellularly replicating bacteria, too. Intracellular efficacy of TB515, PLGA-D1 and PLGA-D2 was evaluated on infected MonoMac6 human monocytes. The infected cells were than treated with TB515, PLGA-D1 and PLGA-D2. After 3 days the treatment was repeated with fresh solution of the compounds for an additional 3 days. Untreated infected cells were considered as negative control.

Compound TB515 was tested on infected MonoMac-6 cells using a concentration of 125 $\mu \mathrm{g} / \mathrm{mL}(25 \times \mathrm{MIC}$ ), and $250 \mu \mathrm{g} / \mathrm{mL}$ (50 x MIC). At these concentrations compound TB515 was able to reduce the number of $\mathrm{CFU}$ compared to untreated control in a concentration dependent manner.

Taking into consideration the drug content of PLGA NPs the final concentration of drug was adjusted to $125 \mu \mathrm{g} / \mathrm{mL}$ in the case of PLGA-D1 and PLGA-D2. Using the PLGA-D1 and PLGA-D2 at $125 \mu \mathrm{M}$ concentration gratifying intracellular inhibition was detected. Significantly reduced CFU was enumerated compared to CFU of the untreated control cells. The TB515 loaded nanoparticles were proved to be more effective than the free compound TB515 since the reduction of the intracellular growth of $M$. tuberculosis achieved by PLGAD1 and PLGA-D2 at $125 \mu \mathrm{M}$ concentration is comparable to reduction obtained by treatment with TB515 at $250 \mu \mathrm{M}$ concentration.

No significant difference was observed between the activity of PLGA-D1 and PLGA-D2.

According to the results, preparation of polymeric nanoparticles has improved the intracellular efficacy of compound TB515 on M. tuberculosis $\mathrm{H}_{37} \mathrm{Rv}$ infected MonoMac-6 cells probably because of the better intracellular availability of the antitubercular. This may be due to the better penetration and cellular uptake rate of nanoparticles into the host cells and better delivery of TB515 to its site of action.

\section{Conclusions}

Surface modification of PLGA nanoparticles where primary amino groups were introduced to the Pluronic surface layer was presented in this work. The nanoparticles showed significant interaction with model membrane system depending on the amount of amphiphilic polymer applied.

An antitubercular drug candidate was encapsulated into the PLGA NPs. This formulation was successfully taken up by cells and enhanced the availability and efficacy of drug which was demonstrated in comparative in vitro experiments.

The PLGA NPs with functionalized surface layer have a great potential as sustained and targeted drug delivery system even at oral or site specific administration like inhalation. This surface modification method provides a platform to achieve the active targeting of such NPs which are sterically stabilized by their surface layer. This seems to be a promising approach since the aggregation stability, the stealth character, and the ability of drug targeting are simultaneously present.

Tuberculosis is still is one of the leading causes of death in the world leading to 8 million infections every year. The antibiotic-loaded nanoparticles could be an effective delivery system to target intracellular infections and to treat or prevent local infections.

It might be another relevant aspect that both PLGA and Pluronic block copolymers are approved by the FDA (Food and Drug Administration) and EMA (European Medicine Agency) in various drug delivery systems [20]. Based on these main components PLGA drug delivery nanoparticles presented here can be in a good position for further development. 


\section{Acknowledgements}

The authors thank Mr.Sándor Dávid and Dr. Nóra Szabó (Laboratory of Bacteriology, Korányi National Institute for Tuberculosis and Respiratory Medicine, Budapest, Hungary) for their participation during the in vitro antimycobacterial evaluation. Valuable assistance of Mrs. I. Hórvölgyi in Langmuir experiments is greatly acknowledged.

This work was supported by the National Science Foundation: OTKA 104928, OTKA 104275 and TÁMOP 4.2.4. A/2-11-1-2012-0001 'National Excellence Program'. 


\section{References}

[1] K.S. Soppimath, T.M. Aminabhavi, A. R. Kulkarni, W.E. Rudzinski, Biodegradable polymeric nanoparticles as drug delivery devices, J. Contr. Rel. 70 (2001) 1-20.

[2] L. Mu, P. H. Seow, Application of TPGS in polymeric nanoparticulate drug delivery system,Coll. Surf. B 47(2006) 90-97.

[3] V.J. Mohanraj, Y. Chen, Nanoparticles - A Review, Tropical J. Pharmac. Res., 5(2006) 561-573.

[4] F. Yan, C. Zhang, Y. Zheng, L. Mei, L. Tang, C. Song, H. Sun, L. Huang, The effect of poloxamer 188 on nanoparticle morphology, size, cancer cell uptake, and cytotoxicity, Nanomedicine 6 (2010) 170-178.

[5] H. M. Redhead, S.S. Davis, L. Illum, Drug delivery in poly(lactide-co-glycolide) nanoparticles surface modified with poloxamer 407 and poloxamine 908: in vitro characterization and in vivo evaluation, J. Contr. Rel. 70 (2001) 353-363.

[6] E. V. Batrakova, A. V. Kabanov, Pluronic block copolymers: Evolution of drug celivery concept from inert nanocarrieres to biological response modifiers, J. Contr. Rel. 130 (2008) 98-106.

[7] É. Kiss, Temperature dependence of BSA adsorption onto polyethylene and PEO-grafted surfaces, Colloids Surf. 76 (1993) 135-140.

[8] S. Park, H. S. Kim, W. J. Kim, H. S. Yoo, Pluronic@Fe3O4 nanoparticles with robust incorporation of doxorubicin by thermo-responsiveness, Int. J. Pharm. 424 (2012) 107-114.

[9] T. I. Croll, A. J. O’Connor, G. W. Stevens, J. J. Cooper-White, Controllable surface modification of poly(lactic-co-glycolic acid) (PLGA) by hydrolysis or aminolysis I: physical, chemical, and theoretical aspects,Biomacromol.5 (2004) 463-473.

[10] É. Kiss, E. Kutnyánszky, I.Bertóti, Modification of poly(lactic/glycolic acid) surface by chemical attachment of poly(ethylene glycol),Langmuir 26(2010) 1440-1444.

[11] É. Kiss, M. G. Takács, I. Bertóti, E. I. Vargha-Butler, Surface properties of poly(lactic/glycolic acid) - Pluronic ${ }^{\circledR}$ blend films, Polym. Adv.Techn. 14 (2003) 839-846.

[12] É. Kiss, A. Varga, E. I. Vargha-Butler, Interfacial behaviour of poly(lactic acid) and Pluronic6400 mixed monolayers at air-water interface, Phys. Chem. Chem. Phys. 6 (2004) 1575-1579.

[13] G. Gyulai, Cs.B. Pénzes, M. Mohai, T. Lohner, P. Petrik, S. Kurunczi, É. Kiss Interfacial properties of hydrophilized poly(lactic-co-glycolic acid) layers with various thicknesses, J. Colloid Interf.Sci. 362 (2011)600-606.

[14] S. Hirsjarvi, L. Peltonen, J. Hirvonen, Surface pressure measurements in particle interaction and stability studies of poly(lactic acid) nanoparticles, Int. J. Pharmaceutics 348 (2008) 153-160. 
[15] T. Trimaille, C. Pichot, A. Elaissari, H. Fessi, S. Briancon, T. Delair, Poly(D,L-lactic acid) nanoparticle preparation and colloidal characterization, Coll. Polym. Sci. 281 (2003) 1184-1190.

[16] D. Jain, R. Athawale, A. Bajaj, S. Shrikhande, P. N. Goel, Studies on stabilization mechanism and stealth effect of poloxamer 188 onto PLGA nanoparticles, Colloids Surf B109 (2013) 59-67.

[17] D. H.Nguyen, J. W. Bae, J. H. Choi,J. S. Lee, K. D. Park,Bioreducible cross-linked Pluronic micelles: $\mathrm{pH}$-triggered release of doxorubicin and folate-mediated cellular uptake, J.Bioact.Compat.Polym. 28 (2013) 341-354

[18] U.S. Toti, B.R. Guru, M. Hali, C.M. McPharlin, S.M. Wykes, J. Panyam, J.A. WhittumHudson, Targeteddelivery of antibioticstointracellularchlamydialinfections using PLGA nanoparticles, Biomaterials 32 (2011) 6606-6613.

[19] É. Kiss, D. Schnöller, K. Pribranská, K. Hill, Cs. B. Pénzes, K. Horváti, Sz. Bősze, Nanoencapsulation of antitubercular drug isoniazid and its lipopeptide conjugate, J. Dispers. Sci. 32 (2011) 1728-1734.

[20] F. Danhier, E. Ansorena, J. M. Silva, R. Coco, A. Le Breton, V. Préat, PLGA-based nanoparticles: an overview of biomedical applications. J. Control. Rel.161(2012) 505-522.

[21] C. Peetla, A. StineV. Labhasetwar, Biophysical interactions with model lipid membranes: applications in drug discovery and drug delivery, Mol Pharm. 6 (2009) 12641276.

[22] R. Horvath, B. Kobzi, H. Keul, M. Moeller, É. Kiss, Molecular Interaction of a New Antibacterial Polymer with a Supported Lipid Bilayer Measured by an in situ Label-Free Optical Technique, Int. J. Mol. Sci. 14(2013) 9722-9736.

[23] A. Hadicke, A. Blume, Interactions of Pluronic block copolymers with lipid monolayers studied by epi-fluorescence microscopy and by adsorption experiments, J. Colloid Interf. Sci. 407 (2013) 327-338.

[24] A. H. Casé, I. P. DallaPicola, M. E. DarbelloZaniquelli, J. C. Fernandes, S. R. Taboga, F. M Winnik,. M. J. Tiera, Physicochemical characterization of nanoparticles formed between DNA and phosphorylcholine substituted chitosans, J. ColloidInterf. Sci.336(2009) 125-133.

[25] C. Peetla, V. Labhasetwar, Effect of molecularstructure of cationicsurfactantsonbiophysicalinteractions of surfactant-modifiednanoparticles with a modelmembrane and cellularuptake, Langmuir 25 (2009) 2369-2377.

[26] T. Kesthelyi, K. Hill, É. Kiss, Interaction of phospholipid Langmuir monolayers with an antibiotic peptide conjugate

J. Phys. Chem. B117(2013)6969-6979. 
[27] É. Kiss, E. T. Heine, K. Hill, Y-C. He, N. Keusgen, Cs. B. Pénzes, D. Schnöller, G. Gyulai, A. Mendrek, H. Keul, M. Möller, Membrane affinity and antimicrobal properties of polyelectrolytes with different hydrophobicity, Macromolec. Biosci. 12 (2012) 1181-1189.

[28] G. Gyulai; Cs. B Pénzes; M. Mohai; F.Csempesz; É. Kiss, Influence of surface properties of polymeric drug delivery nanoparticles on their membrane affinity, Eur. Polym. J. 49 (2013) 2495-2503.

[29] J. M. Harris, E. C. Struck, M. G. Case, M. S. Paley, Synthesis and Characterization of Poly(ethylene Glycol) Derivatives. J. Polym.Sci. Polym. Chem. Ed. 22(2) (1984) 341-352.

[30] K. Horváti, B. Bacsa, N. Szabó, S. Dávid, G. Mező, V. Grolmusz, B. Vértessy, F. Hudecz, S. Bösze, Enhanced Cellular Uptake of a New, in Silico Identified Antitubercular Candidate by Peptide Conjugation,Bioconjug. Chem. 23 (2012) 900-907.

[31] Li C, Palmer WK, Johnston TP: Disposition of poloxamer 407 in rats following a single intraperitoneal injection assessed using a simplified colorimetric assay. J Pharmaceut Biomed 14 659-665 (1996)

[32] L. Sula, WHO Co-operative studies on a simple culture technique for the isolation of mycobacteria. 1. Preparation, lyophilization and reconstitution of a simple semi-synthetic concentrated liquid medium; culture technique; growth pattern of different mycobacteria,Bull. World Health Organ. 29 (1963) 589-606.

[33] L. Sula, WHO cooperative studies on a simple culture technique for the isolation of mycobacteria: 2. Comparison of the efficacy of lyophilized liquid medium with that of Lowenstein-Jensen (L-J) medium,Bull. World Health Organ.29 (1963) 607-625.

[34] H. W. L. Ziegler-Heitbrock, E. Thiel, A. Fütterer, V. Herzog,A. Wirtz, G. Riethmüller, Establishment of a human cell line (Mono Mac 6) with characteristics of mature monocytes, Int. J. Cancer41 (1988) 456-461.

[35] E. L. Wright, D. C. Quenelle, W. J. Suling, W. W. Barrow, Use of Mono Mac 6 human monocytic cell line and $\mathbf{J} 774$ murine macrophage cell line in parallel antimycobacterial drug studies, Antimicrob.Agents Chemother. 40 (1996) 2206-2208.

[36] H. Tomioka, K. Sato, H. Kajitani, T. Akaki, S. Shishido, Comparative antimicrobial activities of the newly synthesized quinolone WQ-3034, levofloxacin, sparfloxacin, and ciprofloxacin against Mycobacterium tuberculosis and Mycobacterium avium complex,Antimicrob.Agents Chemother. 44 (2000) 283-286.

[37] Y. Lin, P. Alexandridis, Temperature-Dependent Adsorption of Pluronic F127 Block Copolymers onto Carbon Black Particles Dispersed in Aqueous Media, J. Phys.Chem. B106 (42) (2002) 10834-10844

[38] É. Kiss, K. Erdélyi, I. Szendrő, E. I. Vargha-Butler, Adsorption and wetting properties of Pluronic block copolymers on hydrophobic surfaces studied by optical waveguide lightmode spectroscopy and dynamic tensiometric method, J. Adhesion 80 (2004) 815-829. 
[39] M. J. Santander-Ortega, A. B. Jódar-Reyes, N. Csaba, D. Bastos-González, J. L. OrtegaVinuesa, Colloidalstability of Pluronic F68-coated PLGA nanoparticles: a variety of stabilisationmechanisms, J. ColloidInterf. Sci. 302 (2006) 522-529.

[40] Z. G. Yue, W. Wei, P. P. Lv, H. Yue, L.Y. Wang, Z.G. Su, G.H. Ma, Surface charge affects cellular uptake and intracellular trafficking of chitosan-based nanoparticles, Biomacromol. 12 (2011) 2440-2446.

[41] B. Novak, C. Astete, C. Sabliov, D.Moldovan, Interaction of PLGA and trimethyl chitosan modified PLGA nanoparticles with mixed anionic/zwitterionic phospholipid bilayers studied using molecular dynamics simulations, APS Meeting 2012, Abs. B41.006 Bull. APS 57, 1 .

[42] T. O. Price, S. A. Farr, X. Yi, S. Vinogradov, E. V. Batrakova, W. A. Banks, A. V. Kabanov, Transport Across the Blood-Brain Barrier of Pluronic Leptin, J. Pharmacol. Exp. Ther.333 (2010) 253-263.

[43] Z. Yang, G. Sahay, S. Sriadibhatla, A. V. Kabanov, Amphiphilic Block Copolymers Enhance Cellular Uptake and Nuclear Entry of Polyplex-delivered DNA, Bioconj. Chem. 19 (2008) 1987-1994. 


\section{Legend to Figures}

Figure 1

Chemical structure of TB515 molecule

Figure 2

AFM images of DPPC+DPPG lipid monolayer before and after penetration of PLGA nanoparticles

Figure 3

Surface pressure of DPPC layer following introduction of bare PLGA ( $\square$ )and Pluronic127amine stabilized PLGA sample3 $(\bigcirc)$ and $6(\nabla)$ NPs into the subphase. Initial surface pressure is $25 \mathrm{mNm}^{-1}$.

Figure 4

Surface pressure of DPPC layer compressed to $15 \mathrm{mNm}^{-1}$ following introduction PLGA NP samples $4(\diamond)$ and $6(\nabla)$ into the subphase.

Figure 5

Surfacepressure of DPPC $(\square)$ and DPPC+DPPG layer $(\bigcirc)$ following introduction of Pluronic127-amine stabilized PLGA NPs (sample 3) into the subphase. Initial surface pressure is $25 \mathrm{mNm}^{-1}$.

Figure 6

Cellular uptake of PLGA-D1 (gray) and PLGA-D2 (black) nanoparticleson MonoMac6 human monocytic cell line using flow cytometry at various drug concentrations. The SD of the data is $\pm 5 \%$.

Figure 7

Fluorescent microscopy images of MonoMac6 human monocytic cells following treatment with PLGA-D1 and PLGA-D2 nanoparticles at two concentrations.

Figure 8

Colony forming unit (CFU) values determined for Mtb infected MonoMac6 human monocytic cells following treatment with pure drug (TB515) and various encapsulated drug formulations (PLGA-D1 and PLGA-D2). The intracellular efficacy was compared to lysed untreated control cells. 